\title{
Propuestas metodológicas para robustecer el conocimiento y entendimiento de la salud mental
}

\author{
El caso de algunos hombres en la paternidad \\ Yeimi Alejandra Colín Paz
}

\begin{abstract}
:
The provision of health services in Mexico has focused mainly on health or, better said, on physical illness and to a lesser extent on the emotional or mental. This document shows the assumptions from which the concepts of health and mental health, the med ical care services and the sources of formal statistical information are built, which offer the inputs for the health authorities to generate health research. Subsequently, two technical alternatives are proposed to position mental health at a level comparable to that of physical health, in order to meet the comprehensive health needs of the Mexican population, although the study subjects who served as an example for the application of these techniques, they were the progenitors of the male gender because they are a segment of the population made invisible by disciplines such as demography and medicine.
\end{abstract}

Keywords:

Methodological Alterative, Health, Mental Health, Disease, Mortality

\section{Resumen:}

La prestación de servicios de salud en México, se ha concentrado principalmente en la salud o mejor dicho en la enfermedad física y en menor medida en lo emocional o mental. En este escrito se documentan los supuestos desde donde se construyen los conceptos de salud y salud mental, los servicios de atención médica y las fuentes de información formal estadística, que ofrecen los insumos para que las autoridades sanitarias generen la investigación en salud. Posteriormente, se proponen dos alternativas técnicas para posicionar a la salud mental en un nivel equiparable al de la salud física, a propósito de atender las necesidades de salud integral de la población mexicana, si bien los sujetos de estudio que fungieron como ejemplo para la aplicación de estas técnicas, fueron los progenit ores del género masculino por ser un segmento de la población invisibilizado por disciplinas como la demografía y la medicina.

\section{Palabras Clave:}

Alternativas metodológicas; Salud; Salud Mental; Enfermedad; Mortalidad.

\section{Introducción}

La Organización Mundial de la Salud (OMS) revela la importancia de la salud mental, como uno de los tres componentes principales del concepto global, que desde 1948 se refiere al "completo estado de bienestar físico, mental y social y no sólo la ausencia de afecciones o enfermedades"(OMS, 2020).

Actualmente, la representación de la salud mental trasciende al concepto de la OMS y se muestra desde el 2015, como el tercero de los Objetivos Globales del Desarrollo Sostenible, "Salud y Bienestar", que forma parte de un esfuerzo entre los líderes mundiales para erradicar la pobreza, proteger al planeta y asegurar la prosperidad (ONU, 2020). De acuerdo con algunas de las metas de este objetivo, se establece como indispensable reducir al 2030 la mortalidad prematura por enfermedades no trasmisibles, mediante el bienestar, la promoción, prevención y el tratamiento a la salud mental; además, fortalecer la prevención y tratamiento para el abuso de sustancias adictivas; $y$ reducir a la mitad, el número de

a Consultora Independiente, https://orcid.org/ 0000-0002-7745-9552, Email: yeimi.colin.paz@ colmex.mx 
muertes y lesiones causadas por accidentes de tránsito (ONU, 2020).

Sin embargo, aunque la salud mental está definida, presente y representada internacionalmente y en nuestro contexto, en el país se destina 2.4 por ciento del gasto público a este rubro (OPS, 2020), a pesar que la OMS recomienda una asignación de entre el 5 y 10 por ciento (Psyciencia, 2020). Así mismo, existe la necesidad de robustecer el conocimiento y entendimiento alrededor de la salud mental, pues los actores involucrados, como el gobierno, las autoridades sanitarias, los prestadores de servicios en salud y los académicos, etc., parecen tener dificultades para saber qué problemáticas atender cuando de salud mental se trata. Dicha contrariedad, se extiende hasta las personas, que de una u otra forma, buscan mantener o mejorar su estado físico (en general) y mental o emocional (en particular); a la par de no saber cómo cuidarse o cómo contribuir a preservar la salud de otros cercanos y miembros de la comunidad.

Por ello, en este documento se reflexiona acerca de la forma en que los conceptos de salud y salud mental, contribuyen en la estructuración del sistema sanitario, los servicios clínicos, el actuar de los prestadores de servicios en salud y en la generación de información formal que sirve como insumo para nutrir las políticas públicas y la investigación en salud. Posteriormente, se expone una mirada alternativa y algunas propuestas metodológicas para conocer y entender algo más de la salud mental.

\section{¿Por qué es importante tener claridad sobre el significado de la salud y la salud mental?}

Las instancias de salud del gobierno mexicano en colaboración con las agencias internacionales como la OMS y la Organización Panamericana de la Salud (OPS), establecen convenios de intervención, tendientes a responder los problemas elementales en términos de salud, pero ¿qué es prioridad?, si parecen atender a una serie de condiciones variadas y quizá distintas a las que necesitan y demandan las personas.

Esto lo podemos observar, en el concepto de salud mental, que en el año 2006 , se sostenía como "el estado de bienestar que permite a los individuos realizar sus habilidades, afrontar el estrés normal de la vida, trabajar de manera productiva y fructífera, y hacer una contribu ción sign ificativa a sus comunidades" (Inmujeres, 2006, p.1); en tanto que en la actualidad, la OMS (2020a) la suscribe como "las actividades de promoción de bienestar, prevención de trastornos mentales y el

\footnotetext{
* Más adelante, se anunciará la razón por la que se cree que hay una relación entre algunos accidentes de tránsito y la salud mental o emocional.
}

tratamiento y rehabilitación de las personas afectadas por dichos trastornos".

No es de extrañar, que los conceptos experimenten ajustes a lo largo del tiempo, pues se espera que cada vez sean más explicativos, efectivos y estables, pero cambiar el sentido de manera radical, parece impráctico, inconsistente e incompatible, si se intenta equiparar en situaciones o contextos particulares en el tiempo. Es decir, la perspectiva de la OMS ha transitado en 2006, de evidenciar a la salud como una capacidad de los individuos en conjunto para generar un bienestar colectivo y productivo en favor del país (como si existiera un cuerpo social humanizado), a sostener en 2020, que no es una cualidad humana, sino una serie de servicios que pueden o deben dotarse mediante el sistema sanitario y acciones de promoción, prevención y atención.

Este escenario de confusión se observa también en el concepto de salud, que si bien no ha cambiado en los últimos setenta años, genera cu estionamientos por ser utópico, idealista y normativo (Caponi, 1997; Alcántara, 2008 y Martínez, 2008), ya que existen diversas discusiones respecto a las valoraciones que se otorga a los componentes del término. Solo por mencionaralgunos ejemplos, hay quienes aluden a la dificultad de medir la salud con base en el "bienestar" (Caponi, 1997 y Le Blanc, 2004), cuya connotación podría ser tan variada como el número de personas que la definan; otros, manifiestan su desacuerdo por intentar aplicar la salud de manera estandarizada, al margen de las diferencias entre los grupos poblacionales 0 de los individuos entre sí (Alcántara, 2008); un punto más, tiene que ver con el contexto histórico (espacio y tiempo) en que se desenvuelven las autoridades médicas en turno y que son quienes determinan qué significa estar sano o enfermo (Alcántara, 2008); además de que pudiera complicarse la identificación de las personas enfermas, debido al periodo de incubación o del tiempo que se tarda una enfermedad en manifestarse en el organismo humano, pues habría quienes no presenten síntomas inmediatos y no por ello estar salu dables (Alcántara, 2008 y Martínez, 2008).

Por otro lado, el concepto de salud, aparentemente, establece el bienestar físico, mental y social al mismo nivel de relevancia; sin embargo, la OMS (que fue la instancia que acuñó el término) no siempre ha puesto el mismo énfasis en los tres elementos. De acuerdo con Pérez (2011), en la década de los años cincuenta y sesenta, este organismo combatía algunas enfermedades específicas y sólo contemplaba marginalmente las condiciones sociales y ambientales; en años posteriores, el modelo neoliberal, desfavorecía a las 
poblaciones con menos recursos económicos y por tanto la OMS, se dio a la tarea de promover los valores de equidad y derechos humanos para conseguir a las personas financiamiento de acceso a los servicios clínicos acorde a su bolsillo (OMS, 2000). Años después, en 2005, cobró mayor importancia el tercero de los elementos de la salud (el bienestar social) y entonces, se promovieron acciones tendientes a definir los determinantes sociales (OMS, 2017). En tanto que, hoy día es una buena oportunidad para traer a cuenta la importancia de la salud mental, cuando los trastornos están afectando a un tercio de la población mundial (Psyciencia, 2020).

\section{La Concepción de Salud y Salud Mental desde el Sistema Sanitario y los Servicios Clínicos}

El sistema de salud mexicano también muestra una falta de equilibrio en términos de la relevancia de los componentes de la salud, pero esto lo enunciaré después de mencionar brevemente la forma en que están estructurados los servicios sanitarios, según las tres etapas descritas por Leal y Martínez (2002):

La primera: está dirigida a ciudadanos sanos, en la que se promueven acciones de promoción a la salud y educación y/o dotación de información para el cuidado.

La segunda: se orienta a usuarios del sistema sanitario, en el que se realizan actividades preventivas con estrategias específicas para combatir ciertas patologías. Por ejemplo: campañas de vacunación, programas de detección de diabetes, hipertensión, obesidad, etc., y;

La tercera: se enfoca en pacientes que presentan patologías o síntomas de las mismas, en donde la oferta de recursos son utilizados para el diagnóstico, tratamiento, rehabilitación y atención de discapacidades, hasta restituir la salud y reintegrar al paciente a su calidad de usuario o ciudadano sano; mientras que el reto para los padecimientos crónicos o discapacitantes no es la cura, sino el cuidado.

Dichas etapas, se alinean a la visión que dicta a la salud como algo proveído por los servicios sanitarios, contemplando la promoción, prevención y atención. Una vez que se cruza la frontera de la tercera etapa (la de atención), la persona se siente enferma y busca apoyo médico, en tanto que el sistema de salud, en el mejor de los casos, lo recibe en un primer nivel de atención (clínica familiar o centro de salud), con un diagnóstico y ofreciéndole tratamiento para su recuperación (Leal y

\footnotetext{
* Los malestares de acuerdo con la visión de Cantoral (2014), son sensaciones subjetivas de padecimientos psíquicos que no se encuentran dentro de los criterios clásicos de salud o enfermedad,
}

Martínez, 2002). Si el médico en este primer nivel determina que requiere de atención más específica o especializada, el paciente es enviado a un segundo nivel, que comprende hospitales generales con especialidades (Leal y Martínez, 2002) en medicina interna, pediatría, ginecoobstetricia, cirugía general y psiquiatría (Vignolo Julio, et. ál., 2011); y finalmente, los casos más graves, con patologías complejas y requerimientos especializados de alta tecnología, se envían a hospitales del tercer nivel de atención (Leal y Martínez, 2002 y Vignolo Julio et. ál., 2011).

En la atención primaria o de primer nivel, aparentemente se resuelve el $85 \%$ de las enfermedades que presenta la población (Vignolo Julio et. ál. 2011), si bien parece ser también el momento en que ocurren los primeros conflictos para la atención a salud mental. Es decir, las personas que buscan mitigar sus malestares ${ }^{*} 0$ dolores físicos (posiblemente asociados a condiciones emocionales o mentales), son recibidas y atendidas por médicos generales, con experiencia escasa o nula en la identificación de síntomas derivados de trastornos psicológicos; además de tener tiempo restringido para sostener una consulta e indagar sobre sus antecedentes personales, familiares y de contexto social que lo rodea y que debieran ser plasmados en el instrumento de detección temprana.

El tiempo promedio porconsulta en el primernivel de atención es de quince minutos (Outomuro y Actis, 2013), en donde el paciente tiene la necesidad de ser escuchado, diagnosticado y tratado médicamente, mientras que el profesional de la salud (médico general) se encuentra preocupado por atenderlo y continuar para cumplir con su cuota de productividad (Rodríguez, et. ál. 2018). Ello, a pesar de existir evidencias que sugieren que las consultas más amplias promueven mejor comunicación entre el médico y el paciente, además que se reconocen los problemas psicosociales y se disminuye el estrés entre ellos, dando oportunidad a que se promueva la salud de mejor manera (Rodríguez, et. ál. 2018).

Este escenario podría complicarse de no realizarse algunos ajustes, debido a que el Programa de Acción Específico de salud mental 2013-2018 ${ }^{\dagger}$, muestra inquietud por continuar preservando y reforzando la atención a la salud mental a este nivel y por este mismo personal médico (SS, 2013). Lo que significaría que el médico general, sin conocimientos o habilidades desarrolladas para identificar los problemas emocionales o mentales, continuaría con la responsabilidad y au toridad para valorarlas clínicamente y decidir sobre la pertinencia

sino que son elementos asociados a nociones tales comoel conflicto o crisis.

† Se consultó el programa más actual dado que no existe uno más reciente. 
o apremio de canalizar al paciente o no, con un profesional de la salud mental y/o al segundo nivel de atención (SS, 2013).

Parecería entonces, que en el primer nivel de atención (clínicas familiares y centros de salud), también se prioriza la condición orgánica o física del individuo, pues en consulta, el médico general no se acompaña por un profesional de la salud mental que apoye la valoración del diagnóstico, con base en la naturaleza de las dolencias o malestares que lleva al paciente a solicitar atención. Ello, podría justificar de alguna forma porqué de cinco personas en México que padecen un trastorno mental, sólo una recibe tratamiento psiquiátrico, y/o también podría explicar, porqué una persona con trastornos mentales visita, en promedio, a cinco médicos antes de poder ser atendido por un profesional de la salud mental (Psyciencia, 2020).

La respuesta inmediata a la ausencia de profesionales de la salud mental a lado de un médico general en el primer nivel de atención, quizá sería la falta de recursos económicos suficientes para robustecer el capital humano. No obstante, se tiene evidencia empírica que muestra que el cincuenta por ciento de los trastornos mentales podrían identificarse a temprana edad y con ello, mejorar la calidad de vida de las personas, al grado de reducir los costos en la atención a la salud, no sólo la mental (Psyciencia, 2020), sino también lo destinado a la reducción del consumo y adicción al alcohol, tabaco y otras sustancias psicotrópicas, a la par de disminuir la cantidad de pérdidas humanas prematuras derivadas de eventos extremos como el suicidio, homicidio y/o accidentes, que en la actualidad, se encuentran posicionadas entre las diez causas de muerte más recurrentes en México (Colín, 2018), por lo que quizá habría que buscar áreas de oportunidad para redirigir los recursos, más que solicitar nuevas partidas.

Por lo dicho hasta ahora, podemos apuntar que el concepto de salud y de salud mental de la OMS, así como el sistema sanitario y los servicios clínicos, muestran una inclinación por preservar un bienestar colectivo a partir de búsqueda del mayor o mejor desarrollo del país, dado que se valora significativamente la calidad y la ampliación de la infraestructura, la productividad institucional y la competencia técnica del capital humano (Figueroa, 1999), que acompaña a los servicios de promoción, prevención y atención.

No obstante, dichos servicios se encuentran condicionados por el crecimiento económico del país y han sido rebasados en cuanto a capacidad, al grado de privilegiar acciones de bajo costo y alto rendimiento, que posicionan al sujeto (ciudadano, usuario o paciente) como responsable de lo que pueda ocurrir con su vida y salud, atribuyéndole el cumplimiento de conductas y prácticas saludables estan darizadas que muchas veces están fuera de sus posibilidades (Leal y Martínez, 2002).

Por dar un ejemplo, pienso en el exhorto de las autoridades en salud para que las personas se alimenten con base en el plato del buen comer y realicen actividad física diaria, sin considerar que una buena parte de las comunidades podrían carecer de acceso a nutrientes esenciales 0 tener mayor facilidad para conseguir alimentos chatarra a menor costo. En tanto que las personas que no hacen ejercicio, podrían privilegiar las amplias jornadas de trabajo o tener más de un empleo para solventar el gasto familiar, y por tal, no valorar su salud por encima del bienestar familiar.

En ambos casos, las personas podrían verse afectados por el estigma o incluso sentir algún tipo de malestar emocional derivado de la imposibilidad o la decisión forzada de no cumplir con las normas establecidas o "sugeridas" por las autoridades de salud o también por el rechazo social, pero aun así, no identificar mejores alternativas. Un caso reciente de descalificación y cuestionamiento colectivo, es salir a la calle y no usar cubrebocas debido a la emergencia sanitaria por COVID19 , sin reflexionar sobre la posible dificultad que tienen, no pocas personas, para dejar de trabajar y conseguir recursos para comer, antes de pensar siquiera en realizar un gasto diario para comprar cubrebocas.

\section{Los supuestos que sustentan la información formal en salud física y mental}

Un último punto a tratar con base en la perspectiva vigente de salud y salud mental, es la información que se produce en el país a propósito de contar con insumos que permitan conocer o ampliar el conocimiento sobre las enfermedades que complican o terminan con la vida de las personas, sobre todo a edades tempranas debido a situaciones relacionadas con la condición psicológica o psiquiátrica.

En un estudio realizado en el 2018, fueron analizados los supuestos desde donde se construyeron cuatro fuentes de información estadística con temas cercanos a la salud física, mental o emocional y de muerte de la población (Colín, 2018). A partir de este, llamó mi atención por un lado, que una de las fuentes que daba bríos sobre la salud mental (aunque no se le llamara así como tal), tenía propósitos diferentes" a la de favorecer las condiciones contextuales, emocionales y de bienestar de la población.

\footnotetext{
* Intentaba ofrecer una herramienta distinta a la información que tienen las agencias internacionales para evaluar de manera complementaria el progreso de la nación.
} 
Por otro lado también descubrí, que los certificados de defunción (que son el instrumentos para generar las estadísticas de mortalidad), son llenados o elaborados por personal médico de la salud física (no mental), y por tal, pudieran estarse subregistrando las muertes que devienen a trastornos mentales, aun y cuando estas enfermedades (las mentales) estén plasmadas en la Clasificación Internacional de Enfermedades (CIE) (Colín, 2018). Por lo que nuevamente se puede sospechar, que en México existe un mayor interés o preocupación por visibilizar, estudiar o incluso combatir las patologías físicas, orgánicas, tangible o probatorias mediante exámenes clínicos, pero no necesariamente, sobre lo que afecta a la población, a las personas en particulary a lo que se tendría que atender para evitar, en la medida de lo posible, las enfermedades y muertes prematuras.

\section{¿Cómo recuperar la importancia de la Salud Mental?}

Una visión distanciada del concepto de salud de la OMS y apegada al pensamiento crítico de Georges Canguilhem", sostienen que la salud no es algo que pueda ser dotado por el sistema sanitario, ni definido por un grupo de autoridades médicas o sanitarias, sino una sensación concebida, experimentada o palpada por el propio individuo. De tal forma que la salud, es la capacidad humana de no estar enfermo, recuperarse de distintas enfermedades $\mathrm{y} / \mathrm{o}$ de adaptarse a vivircon una o varias de ellas, procurando realizar las actividades cotidianas (no sólo las productivas) y evitando deteriorar en la medida de lo posible, el cuerpo biopsicosocial (Le Blanc, 2004; Caponi, 1997 y Martínez, 2008), es decir, esa conformación del cuerpo geno ${ }^{\dagger}$ y fenotipo ${ }^{\ddagger}$ que singulariza nuestras capacidades y que moldea nuestra estructura morfológica (Caponi, 1997).

Igualmente, habría que tener claridad respecto a que la salud es diferente de la enfermedad y que mientras la primera es una capacidad humana, la segunda es la molestia que siente el mismo individuo, que no le permite realizar sus actividades cotidianas y que le hace pensar que hay algo incompatible en la relación que existe entre su cuerpo y el medio en el que se desarrolla (Caponi, 1997 y Le Blanc, 2004), esto además de lo que está más claramente definido en la CIE.

Pero los malestares, dice Cantoral (2014), se encuentran en un lugar intermedio entre la salud y la

\footnotetext{
* Filósofo y médico francés especializado en epidemiología e historia de la ciencia

$\dagger$ Constituido por lo que le es dado genéticamente.

* Que tiene que ver con la forma en la que nos insertamos en el medio, combinando modos de vida elegidos (comportamientos, conductas y toma de decisiones) e impuestos (alimentación
}

enfermedad, por lo que no están definidos como enfermedades aunque afectan a las personas que los experimentan y les generan estados de sufrimiento, aqueján dolas no solo a nivel emocional, sino también a nivel físico. De tal forma, que así como las adversidades contextuales y las enfermedades crónicas pueden afectar el estado emocional de las personas (SS, 2013), los trastornos mentales o desequilibrios emocionales podrían perturbar al estado físico, vulnerando aún más al cueno y promoviendo un espacio propicio para la incubación de diferentes enfermedades o la ocu rrencia de accidentes.

Al ser los malestares elementos intermedios entre la salud y la enfermedad, no son considerados por la OMS ni por las autoridades sanitarias mexicanas como factores de riesgo causantes o determinantes de enfermedades o muerte, por lo que tampoco se encuentran incluidos en los catálogos de enfermedades, y su ausencia remite a su vez a la poca producción de acercamientos teóricos, además de que tampoco están incluidos como materia para ser incluido dentro de los servicios de promoción y prevención para la salud.

Así pues, las acciones implementadas por el gobierno mexicano a partir del sistema sanitario y la prestación de servicios en salud, bajo la guía de este pensamiento crítico, tendrían que estar encaminadas a facilitar al individuo, quien es el agente importante ${ }^{\S}$, las herramientas para lograr mantener o mejorar su calidad de salud, la de sus cercanos y también los no tan próximos pero sí en su entorno (aunque esto último, por complemento y solidaridad, no por obligación o deber social) y para evitar, en la medida de lo posible, ambientes óptimos para el desarrollo de enfermedades físicas y mentales.

De tal forma, que se tendría que fomentar la promoción de la salud; la educación e información sobre salud y enfermedad (física y mental o emocional), así como también la prevención de los malestares, circunstancias o todo aquello que medie o pueda originar una enfermedad. Finalmente, la atención tendría que proveerse para combatir las patologías que el individuo no pudo evitar mediante la promoción y prevención.

Al asumir la guía de pensamiento crítico, se promovería que el sujeto estuviera involucrado e interesado en el tema de su bienestarfísico y mental, al pendiente de tomar decisiones para cuidar de su salud y motivado a desarrollar conductas y comportamientos saludables, pero también acorde a su contexto y necesidades sin que ello represente acciones

deficiente, analfabetismo, escolaridad precaria, distribución desigual de la riqueza, desempleo, etc.).

$\S$ No el desarrollo del país, ni la población como un cuerpo humanizado y tampoco la infraestructura o capital humano de sistema de salud. 
inalcanzables o que lo sometan a una suerte de mayor frustración o estrés, que finalmente, lo supriman en un espacio propicio para desarrollar alguna enfermedad. Además, tendrían que potenciarse las habilidades para conocer el cuerpo, sus emociones y la combinación de estos con el entorno, a fin de que le sea familiar o relativamente sencillo, identificar aquello que le causa conflicto y que requeriría ser transformado desde su persona o atendido por un profesional de la salud física o mental.

En tanto que la función de los profesionales médicos, no sería la de ser autoridad, ni un héroe, sino quien se nutre de lo que solo las personas pueden transmitir respecto a sus malestares o padecimientos físicos y emocionales, quien combina esta información con los conocimientos técnicos de la profesión médica (física o mental) y, quien al final, ofrece opciones $u$ oportunidades de tratamiento, rehabilitación y cuidado; todo ello, en función de tornar al sujeto a su condición original (sana) o a uno de mayor estabilidad y supervivencia, siempre y cuando se respete el derecho del individuo a decidir sobre sí mismo (Le Blanc, 2004; Caponi, 1997 y Martínez, 2008), sin esperar que sea pasivo y obediente a las indicaciones, sino por el contrario, manteniéndose participativo, activo y pendiente de y para sí (para su salud), con sus limitantes pero también con sus virtudes.

De acuerdo con el Programa de Acción Específico de salud mental, las personas que pueden tener un riesgo mayor de sufrir desórdenes mentales son aquellas que se saben con enfermedades crónicas; las expuestas a maltrato, violencia, abandono y discriminación; y aquellas que vivencian desastres naturales, emergencias humanitarias, crisis financieras, problemas familiares, exceso de trabajo, etc. (SS, 2013). Por lo que quizá, cualquier circunstancia podría generarnos inestabilidad psíquica, de no contar con bases sólidas de higiene emocional o mental. La idea sería entonces, que todas esas adversidades contextuales afectaran lo menos posible, pues sería más complicado y costoso intentar erradicar la pobreza, el desempleo, la injusticia, la inseguridad y otros tantos fenómenos existentes.

Las disciplinas en salud tiene en su haber, conocimientos extensos sobre distintas enfermedades físicas y mentales, pero éstas no concentran todo el conocimiento; las condiciones cambian y se encuentran nuevos patrones de comportamiento que promueven patologías antes desconocidas, como el Karojisatsu, que fue definida por la sociedad nipona como el suicidio ejercido por las personas con fatiga crónica (Amagasa, et. ál., 2005); o lo que actualmente es reconocido como el burnout, que igualmente tiene que ver con el creciente esfuerzo de los individuos en el trabajo y que deriva en el desinterés laboral y el agotamiento crónico (Tena, 2014); enfermedad que si bien ya es reconocida, será hasta 2022 cuando se formalice su existencia en la OMS (OMC, 2020). -¿Cómo podríamos abonar información o conocimiento a la salud mental, si las fuentes de información atienden objetivos diferentes que a las necesidades en salud para la población?

\section{Propuestas metodológicas para el estudio de la salud mental}

Hace un par de años, tuve el propósito de indagar alrededor del tema de la salud, enfermedad y muerte de los varones que tenían en común haber vivenciado la paternidad, ello, en virtud de alertar y dialogar acerca de la sobremortalidad prematura de hombres que desde la perspectiva de algunos investigadores y la mía propia, se relaciona con la construcción de la identidad del género masculino (De Keijzer,s/f, Bonino, 2004) y las actitudes o comportamientos adoptados por ellos dentro del espacio de la paternidad (Figueroa, 2011 y Colín, 2018).

Una de las técnicas utilizadas para conocer sobre este tema, fue la implementación de autopsias verbales, que es una herramienta útil para reconstruir la historia de una persona desde que enferma hasta que muere (Lalinde, 2005). El proceso consiste en compilar actas de defunción que declaren las causas de muerte que nos interesa estudiar (muerte materna, homicidio, suicidio, accidente, etc.), para después visitar los hogares de las personas fallecidas y entrevistar al menos a dos miembros, ya sea de la familia o de la comunidad, que hubieran observado incidentes relacionados con la muerte, con el fin de: a) corroborar la información que reposa en la historia clínica; b) obtener información nueva que permita identificar poblaciones y factores de riesgo, y; c) visibilizar fallas en el sistema de salud (Lalinde, 2005 y Gonçalves, et al., 2012).

Esta herramienta no sustituiría al certificado de defunción, que es instrumento utilizado para generar las estadísticas de muerte, entre otras cosas, porque se aplica a grupos pequeños de estudio. No obstante, en aquél estudio que realicé en 2018, resultó de suma utilidad, toda vez que permitió observar dos elementos interesantes que pudieran asociarse a la salud emocional o mental:

El primero es, que no existen elementos suficientes de promoción para la salud de los hombres, y de estos, los que ejercen paternidad, ni tampoco para manejar la presencia constante y repetida de emociones desagradables y hostiles, pero a veces útiles, y en todos los casos, inevitables como el enojo, la presión o estrés, la preocupación, la ansiedad, la impotencia, el temor, la frustración, incertidumbre, mortificación, sentimiento de ser inútil o estar incapacitado, de culpa, vergüenza, 
autodesprecio, etc. Sensaciones que, como anotaba previamente, no son enfermedades, pero que desgastan el cuerpo físico y facilitan el desarrollo de patologías. El segundo, es la presencia de patrones de comportamiento que están asociados con la construcción de género masculino y la paternidad, que pudieron contribuir a mermar la salud de los varones o situarlos en peligro de enfermar, accidentarse, discapacitarse o morir.

Por mencionar solo algunos ejemplos: No mostrar emociones, esconderlas y guardar silencio para evitar que las personas vean sus debilidades, hace que los hombres representen una figura de fortaleza que se espera sean en el imaginario colectivo (Colín, 2018). Pero los silencios, también se relacionan con la presencia de enfermedades cardiacas (Laurence, 2009), y eso no se puede saber a partir de las encuestas de salud o mediante los certificado de defunción, pues ahí no se anota lo que produjo o lo que antecede a la falla cardiaca. La supresión de emociones, convierte a los hombres en una fuente de dolor (Kaufman, 1997) y ello vulnera el cuerpo físico, haciéndolo propicio para desarrollar enfermedades diversas o cometer conductas temerarias. En el caso de los hombres analizados a partir de las autopsias verbales, gustaban de conducir a exceso de velocidad, ingerían alcohol, tabaco, comían de forma desordenada, hacían actividades deportivas de alto impacto y de forma compulsiva, etc (Colín, 2018). Estas conductas, diría Bonino (2004), están asociadas a la identidad de género masculino, no obstante pudieran también ser signos de inestabilidad psíquica o emocional.

Ser proveedor económico único o mayoritario genera tensiones y estrés que acostumbramos a asociar con el trabajo excesivo y por ende con el burnout (Tena, 2014); pero es menos común que lo relacionemos con la paternidad, toda vez que socialmente el hombre es instado a facilitar los recursos económicos para sostener el hogary a los hijos. Para los hombres estudiados a partir de las autopsias verbales, trabajar para sus hijos representaba una tarea fundamental pero estresante; en ocasiones, lo tomaban como una válvula de escape para evadir problemas de tipo familiar y el desempleo era impensable para su propósito como padres (Colín, 2018).

Tampoco recibieron atención a la salud reproductiva, no solo para compartir la responsabilidad con la pareja, sino para evitar dificultades con su propia condición física o emocional (Colín, 2018). Algunos estudios sugieren, que la salud y la vida de los padres expectantes (que vivencian un embarazo junto con su pareja) se encuentra en peligro en diferentes momentos. Por ejemplo, durante el embarazo, momento en que el profesional de la salud ofrece información sobre el proceso que cursará la mujer y el produ cto del embarazo, pero no necesariamente de la forma en que la pareja puede convivir con el embarazo para mantener una relación saludable; o en el parto, al transitar por una serie de emociones extremas positivas (de felicidad y algarabía) y negativas (ansiedad, impotencia, temor, etc.) en un corto periodo tiempo (Machín, 2015) que pudiera alterar su sique; y tampoco posterior al nacimiento, cuando quizá alguno de los miembros de la pareja pudiera verse expuesto a una depresión (Garfield e Isacco, 2009) y no saber cómo actuar o qué hacer ante ello.

Al final, parece continuar predominando la idea generalizada de que para el sistema sanitario, la mujer y el producto del embarazo, son los únicos que ven expuesta su integridad física y vida, además de que es en ellos, en quien se debe concentrar toda la aplicación de recursos y atención, sin considerar la salud emocional y/o mental de la pareja en el proceso, incluso en beneficio del desarrollo apacible del embarazo.

La imposibilidad de convivir con los hijos por separación o divorcio, tener a un hijo enfermo o en peligro de perder la vida, también fue un hecho que pudo haber alterado la salud de los progenitores que fueron sujetos de investigación a partir de las autopsias verbales. Uno de ellos, perdió la vida en el intento por ir a visitar, en otro estado de la república, a sus dos hijos por el cumpleaños de uno de ellos, aun sabiendo que había mal clima por un huracán reciente (Colín, 2018).

Algunos investigadores argumentan que la preocupación, incertidumbre, frustración, mortificación, sentimiento de incapacidad para proteger, culpa, vergüenza, autodesprecio, que se genera por la pérdida de un hijo, promueve escenarios autodestructivos como tener comportamientos temerarios, auto-lesiones, tener relaciones sexuales de riesgo, cometer ilícitos (robar, pelearse, homicidios) para autocastigarse, además de desarrollar cánceres, enfermedades cerebrovasculares, cardiacas o cometer suicidio, etc. (Arditti, et. ál., 2005; Yappert, 2006; Eisensteadt, 2014;Figueroa, 2014; Cacho, 2015).

Evitar asistir a consultas médicas para atender los padecimientos o para el cuidado de la salud, así como automedicarse, fueron otros de los comportamiento presentes en los progenitores que fueron sujetos de estudio mediante las autopsias verbales (Colín, 2018). Condiciones que quizá muestra la necesidad de trabajar más sobre lo emocional o mental, pues de continuar reproduciendo los patrones más acercados al "deber ser hombre o padre" imaginado socialmente, pudiera contribuir a ampliar los casos de enfermedad, discapacidad y muerte a edades prematuras; esto, más allá de pensar en los trastornos ya conocidos e inscritos en la CIE.

La segunda técnica sugerida para lograr robustecer el conocimiento y entendimiento de la salud mental es: expandir la perspectiva de los profesionales de la salud física y mental, dialogando con ellos diversos 
casos paradigmáticos ${ }^{*}$, a fin de motivar el cruce de su conocimiento técnico médico con la reflexión, las dudas, el cuestionamiento a las certezas y finalmente al robustecimiento de los saberes.

En el estudio que realicé hace un par de años y del que he estado haciendo referencia, a los profesionales de la salud física y mental que fueron entrevistados, se les complicaba pensar en la posibilidad de que los progenitores enfermaran o mu rieran por razones distintas a las de los hombres que no ejercían la paternidad, pero tampoco pudieron negar que ocurriera, sobre todo, después de escuchar y analizar con su lente clínico los casos paradigmáticos (Colín, 2018).

Los médicos que de manera resuelta asegu raban que las personas fallecían por factores orgánicos, en tanto que lo "psicológico no mata" (Colín, 2018:241), en algún momento llegaron a reflexionar sobre la posibilidad de requerir más información sobre el estado mental de los difuntos o del entorno en el que ocurrió el suceso de muerte, a fin de tener elementos contundentes para la valoración del deceso. Esta información, decían, pudiera provenir de autopsias verbales, sobre todo en aquellos casos de muerte violenta ${ }^{\dagger}$, que por lo general afectan en mayor medida a los hombres.

El estrés y la depresión fueron componentes a los que recurrieron repetidamente los profesionales de la salud para tratar de explicar los problemas que enfrentaban los progenitores que protagonizaron las historias paradigmáticas (Colín, 2018). Considero que hace falta contar con una mayor imaginación y apertura (sin prejuicios para evitar limitar la expansión del conocimiento) a propósito de nombrar y clasificar la información que se va obteniendo de los propios sujetos respecto de lo que sienten y acuerpan, de modo que cada vez nos sea más familiar y sencillo, reconocer la importancia de lo emocional y mental en la salud y en la vida de las personas. De lo contrario, terminaremos pensando que no existe, o como pasa en la actualidad, que se da mayor visibilidad e importancia a la promoción, prevención y atención de lo físico, como si ello fuera suficiente para mantener una condición saludable de la población.

\section{Reflexiones Finales}

Es importante señalar, que todas las consideraciones que reposan en este documento provienen de una visión enfocada en las ciencias sociales y los estudios de población, no desde disciplinas cercanas a la salud. Sin embargo, contiene elementos útiles para

\footnotetext{
* Fragmentos de la vida de las personas que ofrezcan información para estructurar o relacionar aspectos de la salud física con la emocional o mental.

$\dagger$ Accidentes, suicidios y homicidios.
}

generar un diálogo en favor de la mayor cantidad de involucrados posibles en el tema. Las personas, podrían recibir una mejor calidad en la oferta de servicios de salud integral (física y mental o emocional); los profesionales de la salud física, pudieran liberar carga en el sentido de la responsabilidad, y los profesionales de la salud mental, podrían participar de hacer lo que saben. En tanto que todos participarían de manera activa y coordinada en mantener y mejorar las condiciones de salud de la población.

En este documento, también se ofrecieron elementos para equilibrar el peso de la balanza en favor de la salud mental con respecto de la física u orgánica, pues son ambas las que singularizan y estructuran el cuerpo humano, no así lo social, que requiere de la intervención de sectores distintos al de la salud (aunque la incluye) y cuyos entornos adversos son más difíciles de combatir, al tener menos control sobre ellos.

El eje de análisis en el ejemplo que se utilizó como guía, fueron los progenitores del género masculino. Sin embargo, la salud mental abarca a otros grupos poblacionales que también son afectados por las condiciones actuales de los servicios de salud y por las condiciones perversas de su género, edad, condición económica, cultura, ambiente, etc., por lo que utilizar cualquiera de la propuestas metodológicas sugeridas con distintas poblaciones, podría resultar de lo más enriquecedor e interesante. Esto, además de seguir indagando y procurando posicionar en un lugar representativo o mejor valorado a la salud mental.

Particularmente, me quedo con la inquietud o propuesta de indagar sobre las enfermedades (cardiacas, cánceres, accidentes cerebrovasculares, diabetes, hipertensión), accidentes, suicidios y homicidios que pudieran estar escondiendo padecimientos emocionaleso trastornos mentales y que saturan la incidencia de muertes por causas o determinantes orgánicos, debido al protocolo actualmente establecido para generar las estadísticas mexicanas de mortalidad.

Además, espero poderincidir con esta propuesta, en generar una herramienta del manejo de las emociones y la relación que estas tienen con el sistema endócrino para continuar analizando la forma en que interactúan ambas con el contexto.

Imagino por ejemplo, que la construcción de la masculinidad, acompañada de una producción importante de testosterona y adrenalina, pudieron haber incitado a un grupo de pasajeros de una unidad de transporte público del Estado de México a golpear a un presunto ladrón ${ }^{\ddagger}$, sin

\footnotetext{
* Para mayor información sobre el caso, ver: El Universal versión electrónica:

https://www.eluniversal.com.mx/metropoli/edomex/pasajerosaprovechan-falla-de-delincuente-y-lo-golpean-en-combi visitada el 05 de agosto de 2020.
} 
caer en cuenta que se encontraban expuestos a ser agredidos, morir o a convertirse en delincuentes si mataban a golpes al delincuente, en virtud de sentirse justicieros y aplaudidos por el resto de la sociedad. Sibien, el enojo o la violencia que genera la producción de testosterona acompañada por adrenalina no es la única que interesaría analizar, y tampoco solo en el caso de los hombres. Habría que tenerimaginación teórica para poder establecer patrones de comportamiento relacionados a combinaciones químicas que se generan en el cuerpo y cómo podríamos controlarlos cuando se tornan dañinos o peligrosos para la vida de las personas.

\section{Referencias}

Alcántara, G. (2008). La definición de salud de la Organización Mundial de la Salud y la interdisciplinariedad Sapiens. Revista Universitaria de Investigación, 9(1), 93-107.

Amagasa,T., Nakayama T. y Takahashi Y. (2005). Karojisatsu in Japan: characteristics of 22 cases of work-related suicide. Journal of Occupational Health, 47(2), 157-164.

Arditti, J., Smock, S. y Parkman, T. (2005). It's been hard to be a father: a qualitative exploration of incarcerated fatherhood. Fathering, 3(3), 267-288.

Bonino, L. (2004). Masculinidad, salud y sistema sanitario. El ca so de la violencia masculina, en Ruíz, C. y Blanco, P. (compiladores) La violencia contra las mujeres: Prevención y detección, Madrid: Díaz Santos.

Cacho, L. (2015). Un día normal en un país de sobrevivientes. Ibero-violencia y vida cotidiana, 37, 8-11.

Cantoral, G. (2014). De las desigualdades de Género a los malestares. La cotidianidad de mujeres y varones de San Cristóbal de las Casas, Chiapas. Universidad de Ciencias y Artes de Chiapas.

Caponi, S. (1997). Georges Canguilhem y el estatuto epistemológico del concepto de salud. História, Ciencias, SaludManguinbos, 4(2), 287-307.

Colín, Y. (2018). Salud, enfermedad y muerte de algunos varones que viven o vivieron la experiencia de la paternidad en la Ciudad de México. El Colegio de México.

De Keijzer, B. (s/f). El varón como factor de riesgo: masculinidad, salud mentaly salud reproductiva. Recupera doel 11 de septiembre de 2020

de

http://www.codajic.org/sites/www.codajic.org/files/El\%20varon

$\% 20$ como\%20factor\%20de\%20riesgo 0.pdf

Eisensteadt,E. (2014). Padres de la Plaza de Mayo. Memorias de una lucha silenciosa, Marea Editorial.

Figueroa, J. G. (2014). Yo me quería tirar a abrazar los huesos, Revista Interdisciplinaria de Estudios de Género, 1(2), 197-201. Figueroa, J. G. (2011). Paternidad, mortalidad y salud: ¿es posible combinar estos tres términos? en Estudios sobre Varones y Masculinidades para la generación de políticas públicas y acciones transformadoras. Universidad de la República.71-78.

Figueroa, J. G. (1999). Algunos desencuentros al normar servicios sobre salud reproductiva: un apunte desde la experiencia de organizaciones no gubernamentales. En González, S Organizaciones no gubernamentales que trabajan por la salud reproductiva en México (pp. 139-171). El Colegio de México. Garfield, C. e Isacco, A. (2009). Urban fathers' role in maternal postpartum mental health. Fathering. 7(3). 286-302.

Gonçalves, F., de Souza, M. C.; Meneghel, S.; da Silva, R.; Machado, D.; Conte, M.; Bastos, A. E.; Grubits, S.; Sousa, A. C.; do Nascimento, R.; de Sousa, L. J. y Alves, G. (2012). Autópsia psicológica e psicosocial sobre suicídio de idosos: abordagem metodológica. Ciencia \& Saúde Coletiva. 17(8). 2039-2052.

Instituto Nacionalde la Mujeres (Inmujeres). (2006). Panorama de la salud mental en las mujeres y los hombres mexicanos. Recuperado el 23 de julio del 2020 de $\underline{\text { http://cedoc.inmujeres.gob.mx/documentos download/100779.pd }}$ $\underline{f}$ Kaufman, M. (1997). Las experiencias contradictorias del poder entre los hombres. en Valdés, T. y Olavarría, J. (editores). Masculinidad/es, podery crisis. Flacso. 63-81.

Lalinde, M. I. (2005). La autopsia verbal: reconstruyendo la historia de muerte materna. Secretaría de Salud Alcaldía de Medellín. 45-54. Recuperado de http://medicina.udea.edu.co/Dependencias/Ginecologia/CLAP/pd f/libros/libro 1/laautopsia verbal.pdf

Laurence, J. (2009). Sufrir en silencio puede llevar a un trabajador a la muerte. Periódico La Jornada, miércoles 25 de noviembre de 2009.pp. 2.

Le Blanc, G. (2004). Canguilhem y las normas. Ediciones Nueva Visión.

Leal, G. y Martínez, C. (2002). Cuatro ensayos sobre calidad clínica de la atención. El caso de la salud reproductiva. Serie Académicos CBS, 43. Universidad Autónoma Metropolitana.

Machin, A. (2015). Mind the Gap: The expectation and reality of involved fatherhood. Fathering. 13(1). 36-59.

Martínez, C. (2008). Los múltiples significados de la salud. Un recorrido bajo la guía de Canguilhem. En Martínez, C. (Compiladora), Seis miradas sobre la salud y sus relaciones con el mundo social (pp.35-57). UAM-X.

Orga niza ción Médica Colegial de España (OMC). (2020). La OMS reconoce como enfermedad el burnout o síndrome de estar quemado. Recuperado el 03 de agosto de 2020 de http://www.medicosypacientes.com/articulo/la-oms-reconocecomo-enfermedad-el-burnout-o-sindrome-de-estar-quemado. Organización Mundial de la Salud (OMS). (2020). Concepto de Salud. Recuperado el 23 de julio de 2020 de https://www.who.int/es/about/who-we-are/frequently-askedquestions $\#: \sim:$ text $=\% \mathrm{C} 2 \% \mathrm{BFC} \% \mathrm{C} 3 \% \mathrm{~B} 3 \mathrm{mo} \% 20$ define $\% 20 \mathrm{la} \% 20$ OMS\%20la,ausencia \%20de\%20afecciones\%20o\%20enfermedad es $\% \mathrm{C} 2 \% \mathrm{BB}$.

[ Organización Mundial de la Salud (OMS). (2020a). Concepto de Salud Mental. Recuperado el 09 de julio de 2020 de https://www.who.int/topics/mental health/es/

Organización Mundial de la Salud (OMS). (2017). Determinantes Sociales de la Salud. Recuperado el 31 de enero de 2017 de http://www.who.int/social_determinants/es/

Organización Mundial de la Salud (OMS). (2000). Informe sobre la Salud en el Mundo, 2000. Mejorar el desempeño de los sistemas de salud. 53 ${ }^{\text {a }}$ Asa mblea Mundialde la Salud, 29 de marzo de 2000. Recuperado el 15 de noviembre de 2017 de http://apps.who.int/iris/bitstream/10665/84394/1/sa4.pdf

Organización de las Naciones Unidas (ONU). (2020). Objetivos Globales del Desarrollo Sostenibles. Recuperado el 23 de julio de $2020 \mathrm{de}$ https://www.un.org/sustainable development/es/health/ Organización Panamericana de la Salud (OPS). (2020). OPS insta a aumentar la inversión en salud mental. Recuperado el 31 de julio de

https://www.paho.org/hq/index.php?option=com content $\&$ view= article\&id=14999: mental-health-spending-must-increase-inorder-to-meet-current-needs-in-the-

a mericas\&Itemid $=1926 \&$ lang $=$ es

Outomuro, D. y Actis, A. M. (2013). Estimación del tiempo de consulta ambulatoria en clínica médica. Revista médica de Chile, 141(3). Recuperado el 29 de julio de 2020 de https://scielo.conicyt.cl/scielo.php?script=sci arttext\&pid=S0034 -98872013000300012

Pérez Tamayo,E. M. (2011). Reflexión en torno al artículo: A conceptual framework for action on the social determinants of health. Discussion paper for the commission on social determinants of health. World Health Organization. En Estrada, J. (Edi. Teoría critica de la sociedad y salud pública (pp. 190-199). Universidad Nacional de Colombia y Universidad de Antioquia. Psyciencia. (2020). Datos sobre salud mental. Recupera do el 23 de julio de 2020 de https://www.psyciencia.com/salud-en-mexico2019-a-que-prestar-atencion/

Rodríguez, A., Jarillo E. y Casas, D. (2018). La consulta médica, su tiempo y duración. Medwave2018, 18(5). Recuperado el 28 de julio de 2020 de 
http://www.medwave.cl/link.cgi/Medwave/Enfoques/Ensayos/72

64.act

Secretaría de Salud (SS). (2013). Programa de Acción Específica en Salud Mental. Recuperado el 23 de julio de 2020 de https://www.gob.mx/cms/uploads/attachment/file/11918/Salud

Mental.pdf

Tena, O. (2014). Malestares laborales y condición masculina

Reflexiones en torno a la flexibilidad laboral. En Figueroa J. G.

Políticas públicas y la experiencia de ser hombre. Paternidad, espacios laborales, salud y educación (pp. 51-78). El Colegio de México.

Vignolo J.; Vacarezza, M.; Álvarez, C. y Sosa, A. (2011). Niveles de atención de prevención y atención primaria de la salud. Arch Med Interna. XXXIII (1). 11-14.

Yappert, S. (2006). Vanguardia y retaguardia, Agenda de Mujeres.

El portal de las mujeres argentinas, iberoamericanas y del mercosur. Recuperado el 27 de marzo de 2017 de http://www.agendadelasmujeres.com.ar/index2.php?id=3\&nota $=2$ $\underline{136}$ 